\title{
Recanalização da artéria femoral superficial com stents Zilver: técnica padronizada e análise retrospectiva de 3 anos
}

\author{
Superficial femoral artery recanalization with Zilver stents: \\ standard technique and 3-year retrospective analysis
}

\section{M arcelo Ferreira, Luis Fernando C apotorto, G iafar Abuhadba, M arcelo M onteiro, Luiz Lanziotti*}

\begin{abstract}
Resumo
O bjetivos: D escrever a técnica de recanalização endovascular da artéria femoral superficial e fazer uma análise retrospectiva dos 3 primeiros anos da técnica.

M étodos: Análise retrospectiva dos pacientes tratados entre 2001 e 2004, visando obter as taxas de perviedade das recanalizações. A amostra considerada neste estudo consta de 79 artérias femorais superficiais recanalizadas em 61 pacientes, nos quais foram utilizados exclusivamente a técnica descrita e o mesmo model o destent de nitinol auto-expansível (Zilver, COOK).

Resultados: D os 61 pacientes, 8\% possuíam isquemia crítica de membro inferior e $92 \%$ apresentavam claudicação incapacitante re fratária ao tratamento clínico. A melhora clínica foi observada e referida pelos pacientes numa relação direta à perviedade das recanalizações. A análise estatística demonstrou taxas acumuladas de perviedade primária assistida de 98, 91 e 84\% em 12, 24 e 37 meses, respectivamente. As taxas de perviedade, entendida como fluxo continuado nas recanalizações, foram de 96, 93 e 93\% em 12, 24 e 37 meses, respectivamente.

Conclusões: Consideramos a técnica da recanalização da artéria femoral superficial um método ao mesmo tempo pouco invasivo, com reduzidas complicações e de consideráveis taxas de sucesso anatômico e perviedade, que, em conjunto, são capazes de proporcionar satisfação e qualidade de vida aos pacientes portadores de doença arterial obstrutiva periférica.
\end{abstract}

Palavras-chave: Artéria femoral, angioplastia, stents, claudicação intermitente.

* Serviço Integrado de Técnicas Endovasculares (SITE), Rio de Janeiro, RJ.

Artigo submetido em 21.07.06, aceito em 18.12.06.

J Vasc Bras 2006;5(4):263-70.

Copyright $\odot 2006$ by Sociedade Brasileira de Angiologia e de Cirurgia Vascular.

\begin{abstract}
O bjectives: To describe the endovascular recanalization technique of the superficial femoral artery and perform a 3-year retrospective analysis of the technique.

M ethods: Retrospective analysis of the patients treated between 2001 and 2004, with the aim of obtaining the patency rates of the recanalizations. The sample consisted of 79 recanalized superficial femoral arteries in 61 patients, exclusively using the described technique and the same nitinol self-expanding stent model (Zilver, (OOK).

Results: 0 f the 61 patients, $8 \%$ had critical lower limb ischemia and $92 \%$ had incapacitating claudication refractory to the clinical treatment. Clinical improvement was observed and reported by the patients in a direct correlation with the recanalization patency. The statistical analysis showed accumulated assisted primary patency rates of 98,91 and $84 \%$ in 12, 24 and 37 months, respectively. The patency rates, considered as the continuous flow in the treated area, were 96, 93 and 93\% in 12, 24 and 37 months, respectively.

Conclusions: We consider the recanalization technique of the superficial femoral artery a less invasive method, with few complications and considerable anatomic success and patency rates, which are able of promoting satisfaction and quality of life to patients with peripheral obstructive arterial disease.
\end{abstract}

Keywords: Femoral artery, angioplasty, stents, intermittent claudication.

\section{Introdução}

A última década do século 20 foi certamente 0 período de ouro da cirurgia endovascular, chegando atualmente à correção precisa de diversas patologias arteriais, algumas permitind o o tratamento depacientes de alto risco, consideradosinoperáveis pela via conven- 
cional. G rande parte desta evolução técnica deve-se à criação e aperfeiçoamento constantes dos balões de angioplastia e stents intravasculares. A partir de 1994, com a aprovação pela Food and D rug Administration (FDA) dos stents balão-expansíveis intracoronários, houve largo emprego desses dispositivos, que constam hoje com resultados muito satisfatórios no tratamento da doença aterosclerótica coronariana ${ }^{1-5}$ e, posteriormente, também foram utilizadosno território da artéria femoral superficial (AFS) ${ }^{6}$.

Apósuma angioplastia com balão, éfundamental a compreen são da ocorrência de quatro fatores: a formação de trombos, hiperplasia intimal, remodelamento negativo e o efeito de recoil, ou de mola. O s stents são capazes de eliminar, devido à sua força radial, estes dois últimos ${ }^{7,8}$. A formação de trombos étratada preventivamente com o uso de agentes antiagregantes plaquetários e anticoagulantes. Já a hiperplasia intimal vem sendo tratada com sucesso, no território coronariano, atravésdo emprego dosstentsliberadoresdedroga, que, no território femoral, se encontram em fase de estudo multicêntrico internacional. A hiperplasia intimal consiste basicamente na proliferação de células musculares lisas e deposição de matriz extracelular, em resposta à lesão provocada pela própria angioplastia e à presença do stent em si ${ }^{9-12}$.

U m fator de importância na prevenção da oclusão ou reestenose é a própria estrutura dos stents, baseado em diversos fatores, como o mecanismo de liberação, o material de fabricação, a presença de polímeros e/ ou outras substâncias capazes de induzir inflamação local e outros, tidos como fatores mecânicos. A reestenose induzida pel os fatores mecânicos vem sendo controlada através dos constantes avanços, a cada geração, de novos dispositivos ${ }^{9-12}$, sendo que diversos autores, como $\mathrm{H}_{\text {enry }} 6$ e Scheinert $^{13}$, confirmam haver diferenças mesmo entre modelos distintos de stents auto-expansíveis de nitinol.

\section{M étodos}

Em 2001, após casos iniciais de revascularização endovascular de membros inferiores, padronizamos uma técnica a ser utilizada na recanalização da AFS e artéria poplítea, que passou a ser nossa primeira escolha no tratamento de todos os pacientes com doença arterial obstrutiva periférica (D AOP), claudicação incapacitante e isquemia crítica do membro inferior, sem sucesso terapêutico clínico.
O diagnóstico daD AO P foi realizado com $\mathrm{D}$ oppler e exames clínicos na maioria dos casos. No pósoperatório, ospacientessão submetidosa exameD oppler semestral, ou antes, em caso de piora clínica.

\section{Recanalização da artéria femoral superficial (técnica padronizada pelo SIT E)}

1) Punção femoral retrógrada contralateral.

2) Introdução de bainha $5 \mathrm{~F}$.

3) Introdução de cateter com curva mamária black tip sobreumaguiahidrofílica de $0,035 \times 260 \mathrm{~cm}$ (Road Runner ${ }^{\circledR}$, COOK) para cateterização da artéria ilíaca contralateral.

4) Avanço deste cateter e da guia até a bifurcação femoral contralateral, onde a guia é trocada por uma guia Amplatz reta de 0,035 x $260 \mathrm{~cm}$ para apoiar a troca da bainha curta $5 \mathrm{~F}$ por uma bainha longa aramada $6 \mathrm{~F}$ de $55 \mathrm{~cm}$ (Raabe ${ }^{\circledR}$, COOK).

5) N ovamente, a guia hidrofílica e o cateter de curva mamária são introduzidos por dentro da bainha de $55 \mathrm{~cm}$ e gentilmente avançados em direção à AFS, atéatingir a luz verdadeira da artéria poplítea distal, em alguns casos passando por trajeto subintimal, mas não necessariamente (Figuras 1A e 1B); uma vez que a guia alcança a porção distal da artéria poplítea, a guia hidrofílica é substituída pela guia Amplatz para termos mais suporte e avançar a bainha até a AFS.

6) Tendo o cuidado de manter a guia em posição, 0 cateter é retirado e substituído por um balão de 6 $\mathrm{mm} \times 10 \mathrm{~cm}$ (Pursuit ${ }^{\circledR}, \mathrm{COOK}$ ), que será inflado por cerca de 8 atm em toda extensão da artéria recanalizada, por um período de 3 minutos.

7) M ais uma vez, mantendo-se a guia em posição, o balão é retirado etoda aárea recanalizadaé recoberta com stents, tendo o cuidado de realizar uma sobreposição de pelo menos $10 \mathrm{~mm}$ entreosstents, a fim de evitar que qualquer área entre os stents permaneça descoberta, servindo de estímulo à reestenose (Figura 2).

8) Após controle angiográfico, se necessário, a dilatação é repetida com o mesmo balão e, em seguida, todo material é retirado, seguindo-se selamento arterial percutâneo (Figura 3).

No tratamento clínico pós-operatório, empregamos o regime medicamentoso de ácido acetil salićlico na dose de 325 mg/dia contínua, associado a clopido- 


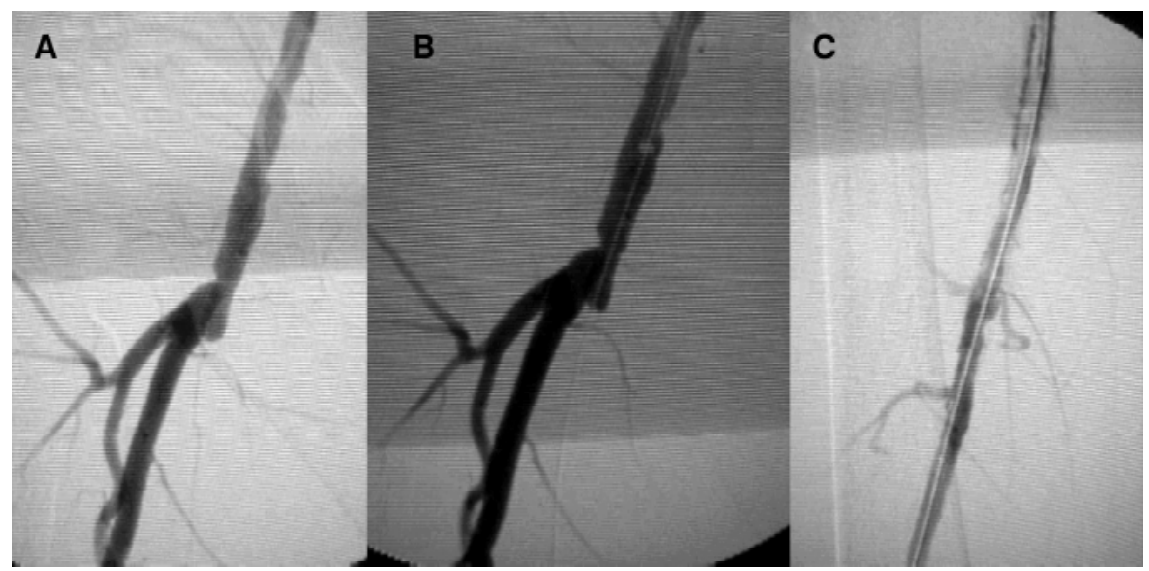

Figura 1 - A) Arteriografia inicial mostrando artéria femoral superficial direita ocluída em sua origem; B) fio guia introduzido na artéria femoral superficial a ser recanalizada; C) angiografia decontrolepós-angioplastia

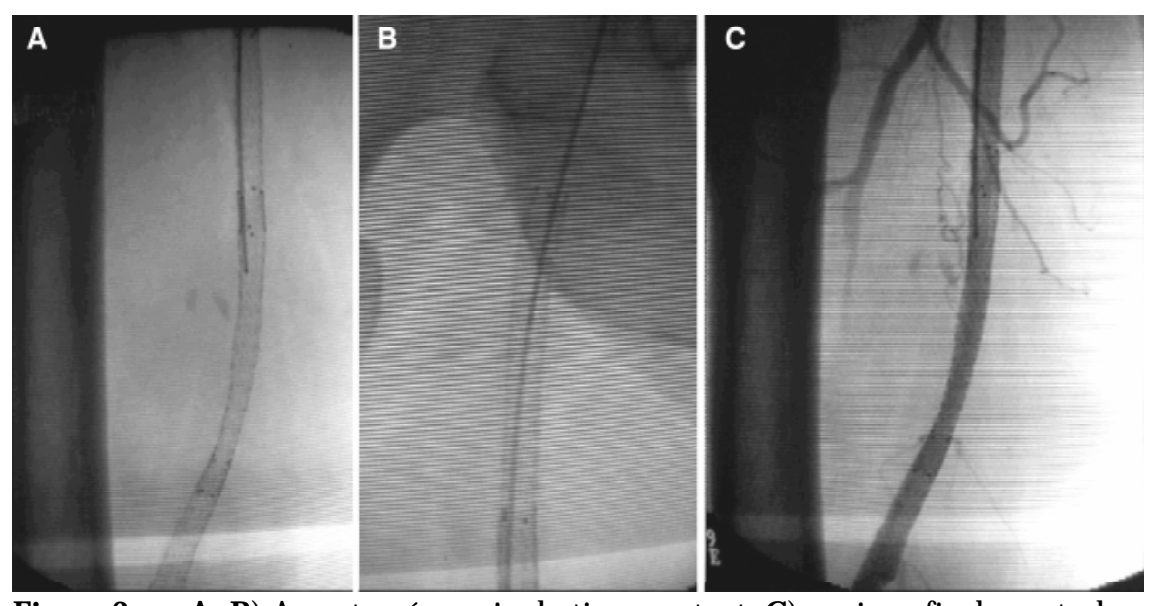

Figura 2 - A, B) Aspecto pós-angioplastia com stent; C) angiografia de controle

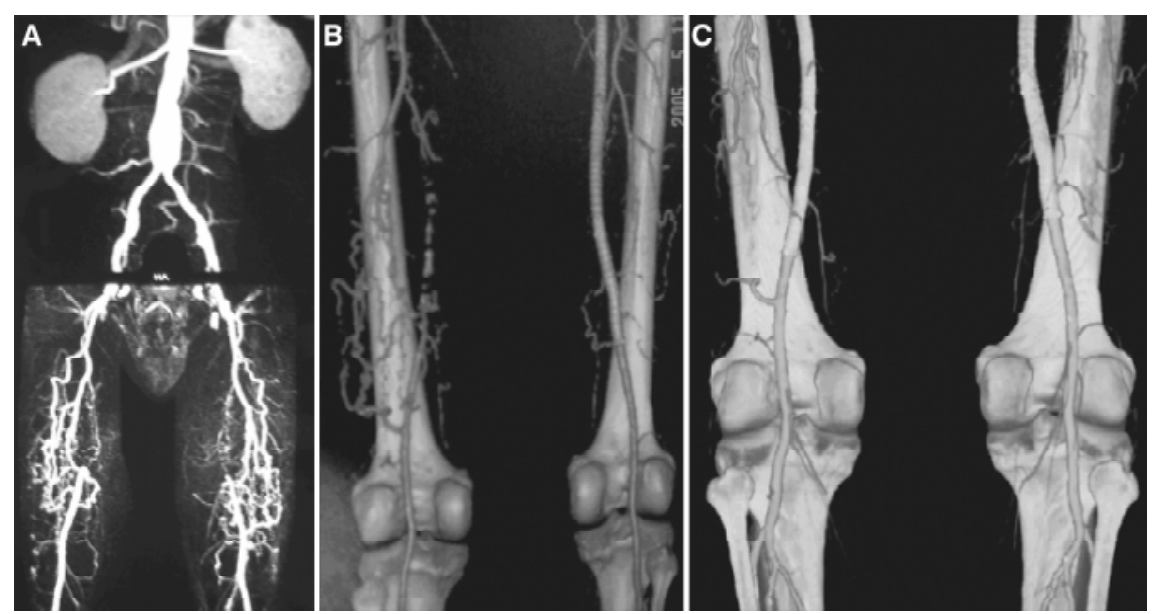

Figura 3 - A) Angiotomografia pré-operatória; B) controle pós-recanalização da artéria femoral superficial direita; C) controle após recanalização da artéria femoral superficial esquerda (notar em B eC os ramos originados no território recanalizado) 
grel na dose de $75 \mathrm{mg} /$ dia por pelo menos 6 meses, visando 0 combate à formação de trombos.

Realiza-se controle pós-operatório com duplex scan em 30 dias e semestralmente após, para acompanhamento da perviedade e detecção de eventuais reestenoses e raio $X$ para detecção de fraturas.

\section{Casuística}

A amostra considerada neste estudo consta de 79 AFS recanalizadas em 61 pacientes, nos quais foram utilizados exclusivamente a técnica descrita e o mesmo modelo de stent de nitinol auto-expansível (Zilver, CO OK). Foram excluídos os pacientes sem acompanhamento clínico adequado e aqueles em que foram empregados outros model os de stents ou técnicas. Sete pacientes (oito AFS) morreram no período deacompanhamento, nenhum deles em decorrência do tratamento aqui discutido, e todos mantinham perviedade primária no momento do óbito.

\section{Análise estatística}

Foi realizada uma análise retrospectiva dos pacientestratadosentre2001 e2004, visando obter as taxas de perviedade e perviedade primária assistida das recanalizações. A data de censura do estudo foi 31/12/2004, e os registros foram divididos em dois grupos para efeito da análise: Grupo 1, considerando os casos em que houve perviedade primária ou primária assistida, quando se detectou estenose maior que $50 \%$ ao D oppler ou piora clínica; Grupo 2, considerando os casos de oclusão dos stents, com ausência de fluxo continuado na área tratada, que revela a taxa de perviedade das recanalizações. N estes pacientes, 0 tratamento empregado para a trombose dos stents consistiu de pontes arteriais, amputações do membro ou, ainda, tratamento clínico.

$\mathrm{N}$ a análise estatística, adotou-se o método de $\mathrm{Ka}$ plan-M eier no cál culo das probabilidades acumuladas e sobrevida e o software SPSS no traçado das curvas de sobrevivência. 0 s resultados foram relatados segundo ospadrões recomendadospel o J ournal of $V$ ascular Interventional Radiology para avaliação clínica de dispositivos de revascularização periférica14.

\section{Resultados}

D os61 pacientes, $8 \%$ possuíam isquemia crítica de membro inferior e 92\% apresentavam claudicação incapacitante refratária ao tratamento clínico. A demo- grafia mostrou ainda que $63 \%$ eram do sexo masculino, $36 \%$ diabéticos, $55 \%$ hipertensos e $72 \%$ relataram tabagismo atual ou no passado. Cerca de $20 \%$ dos pacientes enquadravam-sena classificação T ASC A eB, e os demais nas categorias C eD . O s resultados imediatosmostraram-sesatisfatórios, com sucesso anatômico de $100 \%$ nas recanalizações.

A melhora clínica da claudicação incapacitante foi observada ereferida pelospacientes numa rel ação direta à perviedade das recanalizações.

0 tempo médio de acompanhamento foi de 20 meses(amplitudeentre 0 e 37 meses). $\mathrm{H}$ ouveapenasum caso de trombose aguda, ocorrido no terceiro dia pósoperatório, e $50 \%$ dos pacientes apresentaram mais de 21 meses de acompanhamento ( $P 50=21$ meses), conforme o histograma de sobrevida (Figura 4).

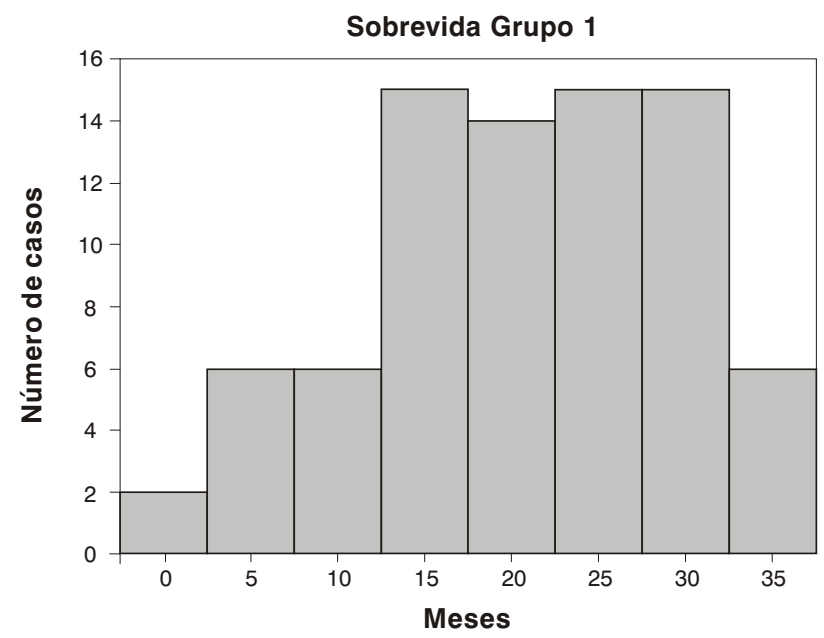

Figura 4 - H istograma de distribuição dos casos acompanhados (em meses) (P25 =15, P50 =21, P75 = 28; média $=20,72 ;$ mediana $=21$ meses)

N este horizonte de 37 meses, cerca de 10\% da amostra $(n=7)$ apresentaram reestenose hemodinamicamente significativa, com obstrução severa ( $>50 \%$ ao D oppler) daluz arterial recanalizada, sendo submetidos a um segundo procedimento endovascular, o que garantiu um sucesso anatômico continuado primário assistido em todos os casos.

D as sete reestenoses, uma ocorreu no mês zero, outra com 7 meses e as demais a partir de 18 meses, 
sendo necessário apenas mais um procedimento em todos os casos. Com o emprego da curva de sobrevivência, obtivemos taxas de perviedade primária assistida acumulada de 98, 91 e 84\% em 12, 24 e 37 meses, respectivamente (Figura 5).

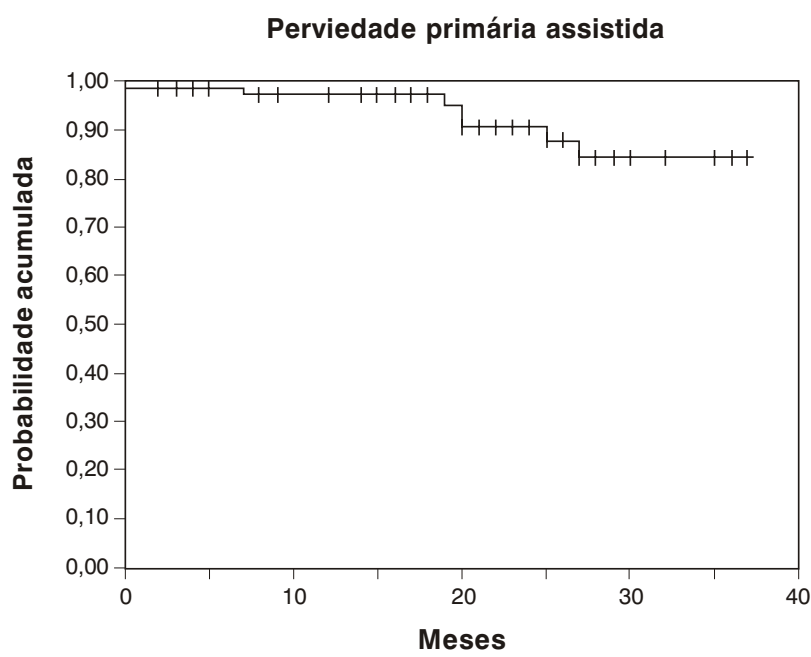

Figura 5 - Perviedade primária assistida acumulada no período de 37 meses entre 2001 e2004, segundo o método de Kaplan-M eier

A distribuição dos casos de reestenose mostrou que $75 \%(n=5)$ dos casos ocorreram entre o 19 e o $27^{\circ}$ mês de acompanhamento, enquanto os demais ocorreram até o sétimo mês (Figura 6).

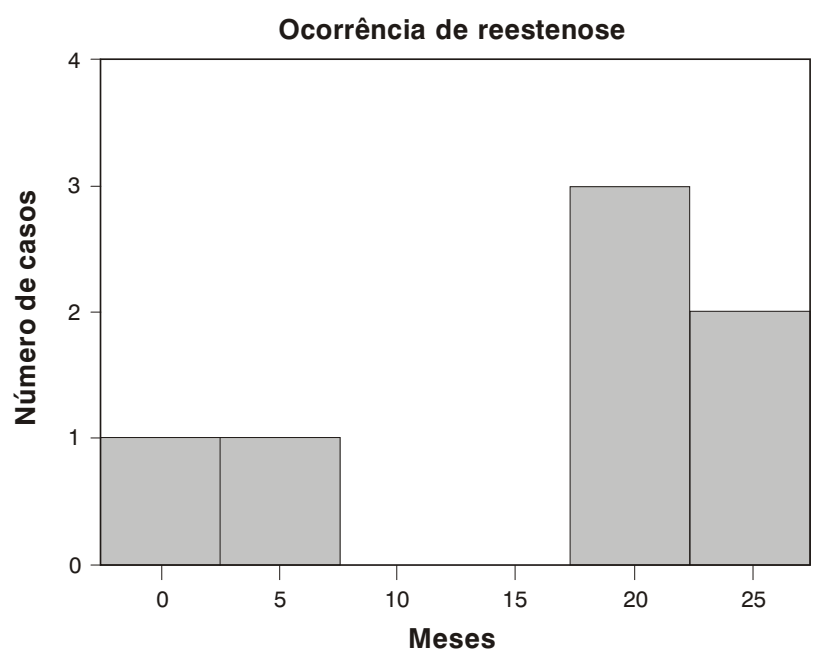

Figura 6 - H istograma de distribuição das ocorrências de reestenose ao longo do período analisado
O s pacientes que sofreram trombose dos stents nas recanalizações de AFS e poplítea não tratada via endovascular representaram cerca de $7 \%$ da amostra $(n=5)$. H ouve uma oclusão no mês zero, duas no quinto mêse duas no $14^{\circ}$ mês de acompanhamento. A curva de sobrevivência em relação a este grupo (Figura 7) demonstra que, ao longo de 37 meses deacompanhamento, as taxas de perviedade, entendidas aqui como presença de fluxo sangüíneo continuado no trajeto recanalizado, foram de 96, 93 e 93\% em 12, 24 e 37 meses, respectivamente. D estes cinco casos, dois foram submetidos a cirurgia de ponte arterial com veia safena, dois tiveram o membro recanalizado amputado e um mantém-se em tratamento clínico, após cura das lesões que indicaram o tratamento inicial.

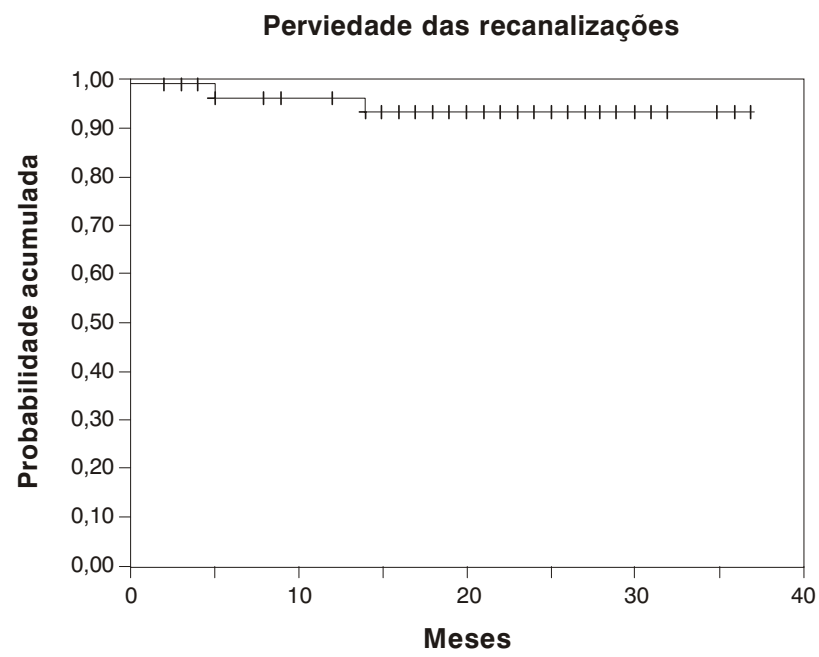

Figura 7 - Perviedade em 37 meses de acompanhamento (notar que a curva se inicia em $98,7 \%$ em função do caso de trombose aguda no pósoperatório imediato)

N os procedimentos primários, utilizaram-se 203 stents de nitinol auto-expansíveis (Zilver, COOK), com média de 2,6 stents por AFS (variação entre um e sete stents). 0 comprimento estimado das recanalizações variou entre 3 e $43 \mathrm{~cm}$, com média de $16 \mathrm{~cm}$ de recanalização na AFS. A análi ise das medidas dos stents demonstra a tendência ao emprego de stents longos, ainda prevalentes em nossa casuística mais recente. Q uanto aos diâmetros, os dados refletem a preferência, nos primeiros meses de uso da técnica, por stents com 
$10 \mathrm{~mm}$ no segmento acima do canal de $\mathrm{H}$ unter e de $8 \mathrm{~mm}$ na AFS abaixo deste e artéria poplítea. I sso não tem mais sido observado em nossa casuística recente, visto quetemos dado preferência aos stents de $8 \mathrm{~mm}$ de diâmetro no segmento da AFS acima do canal de $\mathrm{H}$ unter associados aos de $6 \mathrm{~mm}$ na AFS abaixo deste.

Entreas complicações inerentes à técnica cirúrgica, ocorreram três rupturasfemorais $(2,3 \%)$, tratadas com tamponamento temporário com o próprio baIão de angioplastia; três pacientes $(2,3 \%)$ apresentaram hematoma inguinal, com boa evolução clínica após terapia conservadora e 12 pacientes (9,3\%) apresentaram el evação das taxas de creatinina acima de $1,9 \mathrm{mg} / \mathrm{dl}$ sem, entretanto, haver necessidade de hemodiálise em nenhum desses pacientes, que voltaram a ter os níveis pré-operatórios de creatinina após cerca de 30 dias. Em relação aos stents empregados, houve um caso de fratura macroscópica de uma unidade dos stents, visualizado no raio $X$ de alta definição de controle semestral. Essa fratura não gerou perda da perviedade da recanalização.

\section{D iscussão}

Parece óbvio acreditar que os mesmos resultados obtidos no território coronariano se aplicassem aos demais leitos arteriais, como a AFS. Contudo, ao comparar os diferentes estudos nesses territórios, encontrase grande disparidade na literatura ${ }^{15-20}$.

Acredita-se que isso se deva, em princípio, por ser a AFS mais longa, calibrosa, sujeita a mais pressões, flexibilidade e alterações mecânicas nas diversas direções ${ }^{8}$. 0 utras complicações, como a fratura dos stents, parecem ser mais freqüentes nesse segmento 5 .

Esta controvérsia, entretanto, encontra-se um passo adiante da discussão a respeito de sua aplicabilidade e benefícios, bem definidos em estudos como o SIRO C C O I ell, importantes estudos que analisaram o uso de stents de nitinol no território fêmoropoplíteo ${ }^{5,8}$. N este momento, contamos apenas com os dados de um grande consenso, o T ASC , recomendando a angioplastia fêmoro-poplítea como primeira escol ha nas classes $A$ e $B$, e escolha relativa nas $C$ e $D^{21}$. Contudo, esse consenso já se mostra com defasagem significativa frente à vertiginosa evolução das técnicas e materiais nosúltimos anos. É crescente a opinião de que o TASC merece ser revisado, principalmente no que tange às indicações de angioplastia da AFS, opinião da qual compartilhamos.
$\mathrm{N}$ as lesões descritas neste estudo, a técnica empregada foi a mesma, independente da classificação segundo o TASC. A única influência desta classificação ocorreu em relação às distâncias recanalizadas, sendo estas mais longas e com o emprego de maior número de stents nos pacientes das categorias C e D.

$D$ iversostrabal hos apontam índices promissores de sucesso na angioplastia da AFS, como o de K arch et al., com cerca de $40 \%$ dos pacientes mantendo-se assintomáticos após 4 anos, com possibilidade de reintervenção en dovascular em cerca de $50 \%$ doscasos deinsucesso primário ${ }^{22}$. Como preditores de insucesso imediato, teríamosa idade, a qualidade final da angioplastia (por exemplo, estenose residual, presença de dissecção); em longo prazo (acima de 12 meses), o principal fator de influência na reestenose e oclusão, neste estudo, foi a qualidade final da angioplastia $22-26$.

O TASC comparou 11 estudos referentes à angioplastia com stents no setor fêmoro-poplíteo, em um total de 585 pacientes, eaperviedade primáriamédiafoi de 67\% (variação entre 22 e 81\%) em 23 meses e 58\% em 36 meses. Já os trabal hos referentes apenas à angioplastia, sem uso de stents (oito estudos, 1.241 pacientes), as taxas de perviedade foram de $61 \%$ em 12 meses e $51 \%$ em 36 meses $^{22}$. D eve-seressal tar aqui que, nesses estudos, tanto a técnica empregada quanto os materiais não foram exatamente os mesmos aqui relatados.

U m grande diferencial surgido com os principais estudos multicêntricos sobre o uso de stents na AFS foi a comprovação de que, nesse território, o fator mecânico sobrepõe-se significativamente, a ponto de se julgar que os discrepantes resultados quanto ao grau de reestenose, oclusão, deterioração clínica e fraturas sejam diretamente relacionados ao material e design de fabricação dos stents, assim como suas características físicas, notadamente sua força radial e flexibilidade ${ }^{8}$.

Schlager et al. ${ }^{27}$ avaliaram as taxas de reestenose, deterioração clínica efraturas entre os W al Istents (Boston Scientific) e dois tipos de stents de nitinol autoexpansíveis, SM ART (Cordis) e Dynalink/Absolute (G uidant). T anto para a reestenose imediata quanto para a deterioração clínica, os dados foram favoráveis aos stents de nitinol sem diferenças entre os dois tipos; já quanto à taxa de fraturas, foi significativa a diferença entre os doistipos de stents de nitinol: enquanto a taxa de fratura dos SM ART (Cordis) foi de $28 \%$, no D ynalink/Absolute (Guidant) foi de $2 \%$. Resultados como esses sugerem que, na fase atual do desenvolvimento tecnológico, énecessário avaliar os resultadosindividu- 
ais de cada modelo de stent antes de utilizar um dos diversos disponíveis no mercado.

Ao comparar os resultados obtidos nesta série com outras similares na literatura, buscamos identificar as impressõesclínicas queassociamosàs taxas de perviedade obtidas e ressal tamos aqui al guns pontos relevantes da técnica empregada.

Sem dúvidas, a principal diferença encontra-se na ten dência a realizarmos recanalizações longas e sempre recobrir o território da angioplastia com stents de nitinol auto-expansíveis. Acreditamosque, ao fazer uso dosstents apenas deforma sel etiva nos casos deestenose residual à angioplastia, impede-se apenas 0 efeito de recoil, mais imediato, mas não o remodelamento, queé tardio. O s stents, sendo utilizados sistematicamente, irão permitir a contenção destes dois dos quatro fatores principais de insucesso da angioplastia, citados anteriormente.

A punção femoral homolateral, por diversas razões, como nospacientesobesos, éprejudicada epodeevoluir com complicaçõesobstrutivas exatamentena origem da artéria quesedesejatratar, por isso optamos semprepela punção contralateral. Sefeita homolateralmente, a punção impede a resolução de lesões al tas e aumenta o risco de descolamento de placas e embolia na AFS tratada, além de exigir a compressão ou o uso de dispositivos de fechamento percutâneos, igualmente não isentos de complicações ecom potencial de reduzir o fluxo sangüíneo distal, prejudicando a recanalização arterial. Em nossa experiência, não tivemos complicações decorrentes do emprego da punção contralateral.

D a mesma forma, a experiência no tratamento cirúrgico convencional de artérias femorais mostra-nos que placas ateroscleróticas usualmente tidas como "Iocalizadas" à arteriografia na verdade são muito mais extensas, ea "localização" é nada mais que uma área de maior concentração da estenose. Acreditamos que a recanalização com stents em um trecho longo evita a ocorrência de fraturas nas placas em pontos próximos a esta maior concentração estenótica, que já será foco de maior reação inflamatória após a angioplastia.

É sabido que o local de maior ocorrência de reestenose após angioplastia com stents está nas bordas dos stents. T ambém por essemotivo, noscasosqueapresentam duas ou mais lesões "Iocal izadas", recobrimos todo o território entre os stents, conforme a técnica descrita.

0 stent utilizado nesta série (Zilver, CO O K) possui características específicas de design e resistência em comparação com outros modelos, as quais acreditamos serem responsáveis por parte dos resultados favoráveis. $\mathrm{N}$ otadamente, esse stent possui elevada força radial, talvez por tratar-se de stent utilizado também no tratamento de lesões biliares, o que pode garantir melhor controle do recoil e do remodelamento arteriais. Adicionalmente, o design do stent utilizado traz espaços menoresem sua mal ha, o quepoderia conter a hiperplasia intimal, mas, ao mesmo tempo, permitir o fluxo sangüíneo através de si, mantendo a perviedade dos ramos arteriais originados dos segmentos recanalizados, conforme observamos em diversos exames de controle (Figura 3).

A antiagregação plaquetária e o uso seletivo em al guns pacientes de anticoagulação são outras medidas importantes na manutenção da perviedade, e, acima de tudo, o acompanhamento freqüente dos pacientes com exames clínicos e complementares semestrais permite que se identifiquem precocemente as reestenoses, permitindo melhores resultados em uma segunda recanalização e, principalmente, afastando a ocorrência de oclusões.

N ossa experiência com a técnica de recanalização da AFS mostrou-se muito satisfatória, com taxas de perviedade em longo prazo semelhantes ou superiores às que obtivemos com as pontesfêmoro-poplíteas antes do emprego da técnica endovascular, sem as indesejáveis complicações comuns na cirurgia convencional.

É fato o ceticismo que ainda cerca a técnica de recanalização da AFS, assim como o emprego de stents nestesegmento éum tematalvez controverso. Entretanto, em uma análise ampla da evolução dos dispositivos, notamos firmetendência em favor deambos. Em nossa experiência diária, esta já se tornou a primeira escolha há cerca de 5 anos, gerando benefícios significativos aos nossos pacientes.

\section{Conclusões}

Conformeobservou Palmaz em recenterevisão ${ }^{28}$, é notória a rápida evolução deste campo nos últimos anos, com perspectiva ainda maior para os próximos anos nas áreas de nanotecnologia, microeletrônica e tecnologia de materiais, todos contundentemente envolvidos na engenharia das novas gerações de dispositivos intravasculares. Acompanhando as evoluções tecnológicas, acreditamos que é necessário se adaptar também às técnicas cirúrgicas, exatamente o que tentamos aqui descrever. 
Consideramosa técnica de recanalização da AFS, aqui descrita, um método ao mesmo tempo pouco invasivo, com reduzidas complicações e de consideráveis taxas de sucesso anatômico e perviedade, que, em conjunto, são capazes de proporcionar satisfação e qualidade de vida aos pacientes portadores de DAOP.

\section{Referências}

1. SousaJE, C osta M A, A bizaid AC, et al. Sustained suppression of neointimal proliferation by sirolimus-eluting stents: oneyear angiographic and intravascular ultrasound follow-up. Circulation. 2001;104:2007-11.

2. Sousa JE, Costa MA, A bizaid A, et al. Lack of neointimal proliferation after implantation of sirolimus-coated stents in human coronary arteries: a quantitativecoronary angiography and three-dimensional intravascular ultrasound study. Circulation. 2001;103:192-5.

3. M arx SO, Jayaraman T, Go LO, M arks AR. RapamycinFKBP inhibits cell cycle regulators of proliferation in vascular smooth muscle cells. Circ Res. 1995;76:412-7.

4. Poon M, M arx SO, Gallo R, Badimon JJ, Taubman M B, $M$ arks $A R$. Rapamycin inhibits vascular smooth muscle cell migration. J Clin Invest. 1996;98:2277-83.

5. D uda SH , Pusich B, Richter $\mathrm{G}$, et al. Sirolimus-eluting stents for the treatment of obstructive superficial femoral artery disease: six-month results. Circulation. 2002;106:1505-9.

6. H enry M , K Ionaris C, Amor M, H enry I, T zvetanov K. State of the art: which stent for which lesion in peripheral interventions? T ex H eart Inst J. 2000;27:119-26.

7. H offmann R, M intz GS, D ussaillant RG, et al. Patterns and mechanisms of in stent restenosis: a serial intravascular ultrasound study. Circulation. 1996;94:1247-54.

8. Davies M G, Waldman DL, Pearson TA. Comprehensive endovascular therapy for femoropopliteal arterial atherosclerotic occlusive disease. J Am Coll Surg. 2005;201:275-96.

9. Oliva VL, Soulez G. Sirolimus-eluting stents versus the superficial femoral artery: second round. J V asc I nterv Radiol. 2005;16:313-5.

10. Lee $C, T$ an $H$, Lim $Y$. U pdate on drug-eluting stents for prevention of restenosis. Asian Cardiovasc Thorac Ann. 2006;14:75-82.

11. Duda SH, Poerner TC, W iesinger B, et al. D rug-eluting stents: potential applications for peripheral arterial occlusive disease. J V asc Interv Radiol. 2003;14:291-301.

12. H eldman AW, Cheng $L$, Jenkins $G M$, et al. Paclitaxel stent coating inhibits neointimal hyperplasiaat 4 weeksin aporcine model of coronary restenosis. Circulation. 2001;103:2289-95.

13. Scheinert D, Scheinert S, Sax J, et al. Prevalence and clinical impact of stent fractures after femoropopliteal stenting. J Am Coll Cardiol. 2005;45:312-5.

14. SacksD, M arinelli D L, M artin LG, et al. Reporting standards for clinical evaluation of new peripheral arterial revascularization devices. J Vasc Interv Radiol. 2003;14: S395-404.
15. H ong M K, Kornowski R, Bramwell O, Ragheb AO, Leon M B. Paclitaxel-coated Gianturco-R oubin II (GR II) stents reduce neointimal hyperplasia in a porcine coronary in-stent restenosis model. Coron Artery Dis. 2001;12:513-5.

16. Z danowski Z, Albrechtsson U, L undin A, et al. Percutaneous transluminal angioplasty with or without stenting for femoropopliteal occlusions? A randomized controlled study. Int Angiol. 1999;18:251-5.

17. V roegindeweij D, Vos LD, T ielbeek AV, Buth J, vd Bosch $\mathrm{HC}$. Balloon angioplasty combined with primary stenting versus balloon angioplasty alone in femoropopliteal obstructions: A comparative randomized study. Cardiovasc Intervent Radiol. 1997;20:420-5.

18. Chatelard P, G uibourt C. Long-term results with a Palmaz stent in the femoropopliteal arteries. J Cardiovasc Surg (Torino). 1996;37(3 Suppl 1):67-72.

19. Sapoval M R, Long AL, R aynaud AC, Beyssen BM , Fiessinger JN, Gaux JC. Femoropopliteal stent placement: long-term results. Radiology. 1992;184:833-9.

20. Liermann D, Strecker EP, Peters J. The Strecker stent: indications and results in iliac and femoropopliteal arteries. Cardiovasc Intervent Radiol. 1992;15:298-305.

21. Strecker EP, Boos IB, Gottmann D. Femoropopliteal artery stent placement evaluation of long-term success. Radiology. 1997; 205:375-83.

22. TASC working Group. -M anagement of peripheral arterial disease(PAD ) T ransAtlantic Inter-Society Consensus(T ASC ). Eur J Vasc Endovasc Surg. 2000;19 Suppl A:1-244.

23. K arch $L A, M$ attos $M A, H$ enretta JP, M CLafferty RB, Ramsey $D E, H o d g s o n ~ K J$. Clinical failure after percutaneous transluminal angioplasty of thesuperficial femoral and popliteal arteries. J V asc Surg. 2000;31:880-7.

24. V roegindeweij $D, T$ ielbeek AV, Buth J, van K intsM J, Landman GH, M ali WP. Recanalization of femoropopliteal occlusive lesions: a comparison of long-term clinical, color duplex US, and arteriographic follow-up. J V asc Interv Radiol. 1995;6: 331-7.

25. Surowiec SM, D avies M G, Eberly SW, et al. Percutaneous angioplasty and stenting of the superficial femoral artery. J V asc Surg. 2005;41:269-78.

26. el-Bayar H, Roberts A, H ye R, D avis G, Freischlag J. $D$ eterminantsof failurein superficial femoral arteryangioplasty. Angiology. 1992;43:877-85.

27. Schlager $O, D$ ick $P$, Sabeti $S$, et al. Long-segment SFA stenting-the dark sides: in-stent restenosis, clinical deterioration, and stent fractures. J Endovasc Ther. 2005;12: 676-84.

28. Palmaz JC. Intravascular stents in the last and the next 10 years. J Endovasc Ther. 2004;11 Suppl 2:II200-6.

Correspondência:

M arcelo Ferreira

Rua Siqueira Campos 59/203 - Copacabana

CEP 22031-070 - Rio de Janeiro, RJ

Tel.: (21) 2236.1637

E-mail: mmvf@uol.com.br 